\title{
Fault detection and isolation in flat navigation canals
}

\author{
P. Segovia ${ }^{1,2}$, L. Rajaoarisoa ${ }^{1}$, \\ E. Duviella ${ }^{1}$ \\ ${ }^{1}$ Mines-Douai IA, France \\ Email: \{pablo.segovia, lala.rajaoarisoa, \\ eric.duviella\}@mines-douai.fr
}

\author{
J. Blesa ${ }^{3}$, F. Nejjari ${ }^{2}$, V. Puig ${ }^{2,3}$ \\ 2 Automatic Control Department \\ Technical University of Catalonia (UPC) \\ ${ }^{3}$ Institut de Robòtica i Informàtica \\ Industrial (CSIC-UPC) \\ Email: \{joaquim.blesa,fatiha.nejjari, \\ vicenc.puig\}@upc.edu
}

\author{
K. Horváth \\ Department of Mechanical Engineering \\ Eindhoven University of Technology \\ The Netherlands \\ Email: k.horvath@tue.nl
}

\begin{abstract}
Inland navigation networks are composed of several artificial canals that are characterized by no slope. These canals are particularly subject to resonance phenomena, which can create waves such that the navigation condition might not be guaranteed. It is therefore required to ensure dealing with freefault measured data and actuators. In this work, a fault detection and isolation method based on the Integrator Delay Zero model (IDZ) is designed for flat navigation canals. The proposed method is dedicated to the detection and isolation of sensor and actuator faults. Finally, it is tested by considering the Cuinchy-Fontinettes canal located in the north of France.
\end{abstract}

\section{INTRODUCTION}

Inland navigation systems are large-scale networks composed of natural rivers and artificial canals. Natural rivers are usually canalized, and just like artificial canals, they are equipped with locks that enable the navigation. A navigation reach is defined as a portion of a canal between two locks, whose operation can cause high magnitude waves that reflect from downstream to upstream during several hours, which is known as the resonance phenomena. This situation is especially critical for those reaches that are characterized by no slope (otherwise known as flat reaches).

The dynamics of a navigation reach can be accurately modeled by the Saint-Venant partial differential equations [1]. However, these equations have no known analytical solution, which is one of the reasons why simplified models have been developed [2]. The Integrator Delay model proposed by [3] was adapted by using an additional zero in the model leading to the Integrator Delay Zero model (IDZ) [4]. The Integrator Resonance model (IR) developed by [5] is dedicated to freesurface water systems whose dynamics are characterized by the resonance phenomena. Finally, gray-box models have been proposed in [6], [7] to deal with navigation reaches for which the physical parameters are not well known. Among these different options, the IDZ model was used in [8] to diagnose sensor faults in the Cuinchy-Fontinettes reach (CFr), which is located in the north of France. Fault diagnosis is an important issue for inland navigation systems because an error provided by a level sensor could lead to navigation stoppage [?]. Indeed, navigation is allowed only if the level of each navigation reach is inside a navigation rectangle, which is defined by two boundaries of several centimeters around the Normal Navigation Level (NNL). If the water level crosses one of these boundaries, the navigation has to be stopped.

In [9], the diagnosis approach based on IDZ was combined with a pattern recognition method with the objective to benefit from both methods. The IDZ model (which corresponds to the nominal model) and the intervals outputs provided by the interval model are used as features for the classification algorithm. The combination of these two approaches allows the detection and isolation of sensor faults. A similar approach based on gray-box models was proposed in [10].

In this paper, a sensor and actuator fault diagnosis method based on an IDZ model is proposed. The modeling step leading to the estimation of the IDZ parameters is achieved by considering specific characteristics of navigation reaches without slope. Hence, only the backwater dynamics are considered. Then, a fault diagnosis approach is designed to detect and isolate sensors and actuator faults. The proposed methodology is illustrated by the case study.

The structure of this paper is as follows: the case study, the management objectives and the constraints on navigation conditions are presented in Section 2. The IDZ modeling step is described in Section 3. Section 4 is dedicated to the presentation of the fault diagnosis method. Finally, faulty scenarios are simulated using the case study to highlight the performance of the proposed sensor and actuator fault diagnosis methodology.

\section{PROBLEM STATEMENT}

The inland navigation network in the north of France is the largest in the country. It is composed of more than 50 navigation reaches connected through locks. Some of these navigation reaches are artificial canals, which is the case of the CFr.

This navigation reach is equipped with the lock of Cuinchy at the upstream end and with the lock of Fontinettes at the downstream end. A lock operation in Cuinchy corresponds to an exchanged water volume of $3,700 \mathrm{~m}^{3}$, whereas a lock operation in Fontinettes entails $25,000 \mathrm{~m}^{3}$. In order to balance the exchanged water volumes during navigation periods, the $\mathrm{CFr}$ has been equipped with a controlled gate in Cuinchy, which is used to supply the CFr. The daily period of navigation corresponds to 14 hours, starting from 6 a.m.

The CFr is characterized by no slope, a length of 42,3 $\mathrm{km}$, a width of $52 \mathrm{~m}$ and a complex geometry, which can be simplified by considering an average rectangular profile (see Fig. 1).

The management of the CFr consists in maintaining the water level within 3.8 (i.e. the NNL) $\pm 0.05 \mathrm{~m}$. These 


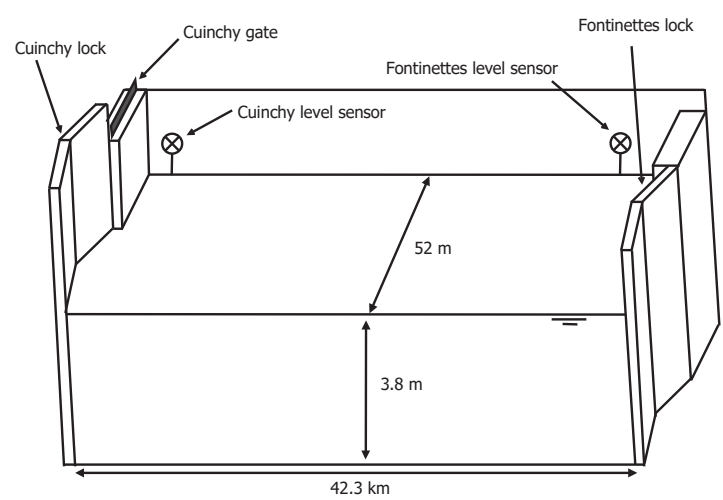

Fig. 1: Schematic view of the CFr.

boundaries correspond to the navigation rectangle. If the water level crosses the navigation rectangle, the navigation has to be stopped. These strong constraints on the water levels require the implementation of efficient control algorithms and fault diagnosis methodologies.

Thus, a fault diagnosis method is designed to detect and isolate faults that can occur on the Cuinchy and Fontinettes level sensors and on the Cuinchy gate. This method is based on the IDZ model of the CFr. The modeling step of free-surface water systems without slope is presented in the next section.

\section{MODELING OF FLAT NAVIGATION CANALS}

As it has already been stated, several simplified models can be used to describe the dynamics of flat canals. Among all the existing options, the IDZ model is used in this work due to its simplicity and its capability to characterize the system dynamics with high accuracy in all regimes. It consists of an integrator, a delay and a zero: while the two first terms reproduce the low frequencies behavior, the zero accounts for the high frequencies. Its structure is as follows:

$$
p_{i j}(s)=\frac{\alpha s+1}{\mathcal{A} s} e^{-\tau s},
$$

where $\alpha$ represents the inverse of the zero, $\mathcal{A}$ the integrator gain and $\tau$ the propagation time delay. The exact values of these parameters cannot be obtained, but they can be precisely approximated as shown in [4]. As the used parameters are an estimation of the theoretical ones, the notation $\hat{p}_{i j}$ replaces $p_{i j}$ hereinafter.

The integrator gain represents how the volume changes depending on the variation of the water level. The time delay constitutes the minimum time that a perturbation needs to travel from its origin to the measurement points. Two different time delays have to be computed, one for the propagation from upstream to downstream of the pool and another one from downstream to upstream. Finally, the zero approximates through a constant gain the oscillatory phenomena that occurs in high frequencies.

These parameters are computed for both the upstream uniform and the downstream backwater part of the canal, and they are then merged into the so-called equivalent parameters, which describe the whole pool.
Finally, the transfer function matrix that links the measured water depths and the discharges at the ends of a canal is given by:

$$
\left[\begin{array}{l}
y(0, s) \\
y(L, s)
\end{array}\right]=\underbrace{\left[\begin{array}{ll}
\hat{p}_{11}(s) & \hat{p}_{12}(s) \\
\hat{p}_{21}(s) & \hat{p}_{22}(s)
\end{array}\right]}_{\boldsymbol{P}(s)}\left[\begin{array}{c}
q(0, s) \\
q(L, s)
\end{array}\right],
$$

where 0 and $L$ are the abscissas for the initial and final ends of the canal; $y(0, s)$ and $y(L, s)$, the upstream and downstream water levels; $q(0, s)$ and $q(L, s)$, the upstream inflow and downstream outflow; and $\hat{p}_{i j}$, the different IDZ terms presented in (1), respectively.

The CFr is a flat navigation canal, which means that it is completely under backwater flow. Therefore, the equivalent parameters are not computed but the backwater parameters are used to describe the whole canal, yielding the following IDZ transfer functions:

$$
\begin{gathered}
\hat{p}_{11}=\frac{6928 s+1}{2.2 \cdot 10^{6} s} \\
\hat{p}_{12}=\frac{-9544 s-1}{2.2 \cdot 10^{6} s} e^{-6930 s} \\
\hat{p}_{21}=\frac{9544 s+1}{2.2 \cdot 10^{6} s} e^{-6920 s} \\
\hat{p}_{22}=\frac{-6928 s-1}{2.2 \cdot 10^{6} s}
\end{gathered}
$$

These transfer functions are obtained by considering an average discharge of $0.6 \mathrm{~m}^{3} / \mathrm{s}$ and a Manning coefficient equal to 0.035 .

These results are compared with a reference model that comes from the solution provided by thye hydraulic simulator SIC $2^{1}$ [11]. The obtained IDZ model predicts the correct downstream water level, but it is not the case for the upstream water level. This mismatch in the estimation of the upstream water level is corrected by means of the following calibration strategy:

$$
\hat{p}_{i j}^{\prime}(s)=\frac{k \cdot \alpha s+1}{\mathcal{A} s} e^{-\tau s},
$$

where $k$ is the calibration coefficient. Notice that only the zeros are calibrated: indeed, the mismatch between the reference and the IDZ model mainly affects the peak response, for which the zero accounts. Therefore, this calibration aims at reproducing the peak magnitude with more accuracy.

The models are calibrated for the Fontinettes lock operation, as it involves the largest water volume exchange between reaches. The calibrated IDZ model for the upstream water level is:

$$
\begin{gathered}
\hat{p}_{11}^{\prime}(s)=\frac{5995 s+1}{2.2 \cdot 10^{6} s} \\
\hat{p}_{12}^{\prime}(s)=\frac{-7003 s-1}{2.2 \cdot 10^{6} s} e^{-6930 s}
\end{gathered}
$$

In addition, $\hat{p}_{21}^{\prime}(s)=\hat{p}_{21}(s)$ and $\hat{p}_{22}(s)=\hat{p}_{22}^{\prime}(s)$.

${ }^{1}$ http://sic.g-eau.net/ 
Figure 2 shows the evolution of the water level at the upstream and downstream ends for the reference, the original uncalibrated IDZ model (3) and the calibrated IDZ model (5). It can be seen that the calibration strategy enables a more accurate prediction of the upstream water level.

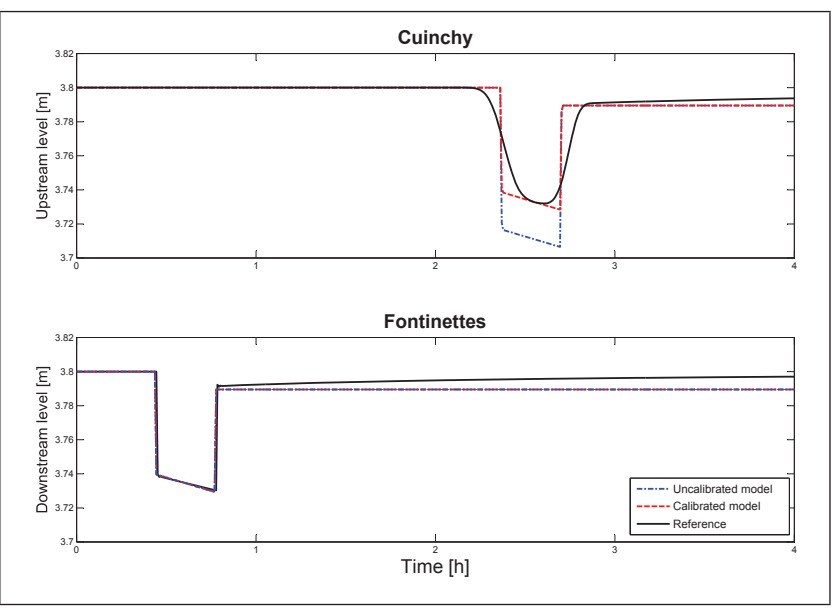

Fig. 2: Evolution of the water levels for the Fontinettes lock operation.

\section{FAULT DIAGNOSIS}

\section{A. Fault detection}

The principle of model-based fault detection is to test whether the measured inputs and outputs from the system lie within the behavior described by a model of the faultless system. If the measurements are inconsistent with the model of the faultless system, the existence of a fault is proved. A deep knowledge of the physical principles that take part in the system is required to compute a model that represents the normal behavior of the plant. This model can describe the behavior of the system in any non-faulty scenario and in the faulty scenarios where the fault can be modeled by a change of model parameters or variables. This does not happen in data driven approaches, where their applicability is restricted by the available non-faulty and faulty data.

In general, two different types of models can be distinguished: qualitative and quantitative models. Quantitative models are used in the Systems Dynamics and Control Engineering community [12], [13], [14] known as FDI (Fault Detection and Isolation) community. Quantitative models are mathematical models that can be described in time or frequency domain. Most of the fault detection techniques based on this kind of models use a temporal residual $r(t)$ that describes the consistency check between the predicted $\hat{y}(t)$ (given by the model) and the real behavior $y(t)$ measured by a sensor.

$$
r(t)=y(t)-\hat{y}(t)
$$

This fault detection approach is based on analytical redundancy.

Ideally, in quantitative model-based fault detection methods, residuals should only be affected by the faults. However, the presence of disturbances, noise and modeling errors causes residuals to become nonzero in the absence of faults and thus interfering with the fault detection. Therefore, the fault detection procedure must be robust against these undesired effects [15].

One of the most developed robust FDI approaches, called active, is based on generating residuals which are insensitive to uncertainty and at the same time sensitive to faults [15]. For instance, designing a filter $G_{f}(s)$ in such a way

$$
\left\{\begin{array}{l}
G_{f}(s) R(s)=0 \text { in a fault-free scenario } \\
G_{f}(s) R(s) \neq 0 \text { in a faulty scenario }
\end{array}\right.
$$

where $R(s)=\mathscr{L}\{r(t)\}$ and $\mathscr{L}\{\}$ is the Laplace transform.

In practice, the perfect decoupling is not possible and $G_{f}(s) R(s)$ can become nonzero even in the absence of faults. On the other hand, in passive robust approaches the model errors are propagated to the residuals, and if the observed residual is not consistent with the model uncertainty, a fault is determined. One way of implementing the passive robust fault detection is to compute the maximum positive and negative deviations (bounds $\underline{\sigma}$ and $\bar{\sigma}$ ) of the residual $r(t)$ in the time domain from zero in a fault-free scenario; therefore, the values of the bounds are directly linked to the accuracy of the model. Then, the following fault detection test can be formulated:

$$
\left\{\begin{array}{l}
r(t) \in[\underline{\sigma}, \bar{\sigma}] \Rightarrow \text { No Fault }(\phi(t)=0) \\
r(t) \notin[\underline{\sigma}, \bar{\sigma}] \Rightarrow \text { Fault }(\phi(t)=1)
\end{array}\right.
$$

On the other hand, faults that produce a residual deviation smaller than the threshold will not be detected (missed alarms), which means that a minimum fault magnitude is necessary to guarantee the fault detection. According to [12], the minimum detectable fault corresponds to a fault that brings a residual to its threshold (triggering limit), assuming that no other faults and nuisance inputs are present.

\section{B. Fault isolation}

Detecting faults is possible with only one residual sensitive to all faults. However, fault isolation usually requires the evaluation of a set of fault signals $\phi_{1}, \ldots, \phi_{n_{r}}$ computed by means of (8) applied to a set of residuals $r_{1}, \ldots, r_{n_{r}}$. The fault isolation module used in this paper derives from the ones proposed in [16] and [17] (see Fig. 3). The first component is a memory that stores information about the fault signal occurrence history and the fault detection module updates it cyclically. The pattern comparison component compares the memory contents with the stored fault patterns. The standard Boolean fault signature matrix concept [12] is generalized taking into account more fault signal properties. The last component represents the decision logic part of the method which aim is to propose the most probable fault candidate.

\section{Memory component}

The memory component consists of a table in which events in the residual history are stored. For each row, the first column stores the event occurrence time $t_{0}$ and the second one stores the maximum activation value $\phi_{i, \max }$

$$
\phi_{i, \max }=\max _{t \in\left[t_{o}, t_{o}+T_{w}\right]}\left|\phi_{i}(t)\right|
$$

for every fault signal $\phi_{i} i=1, \ldots, n_{r}$. Using this strategy, the effect of noise and non-persistent fault indicators are filtered 


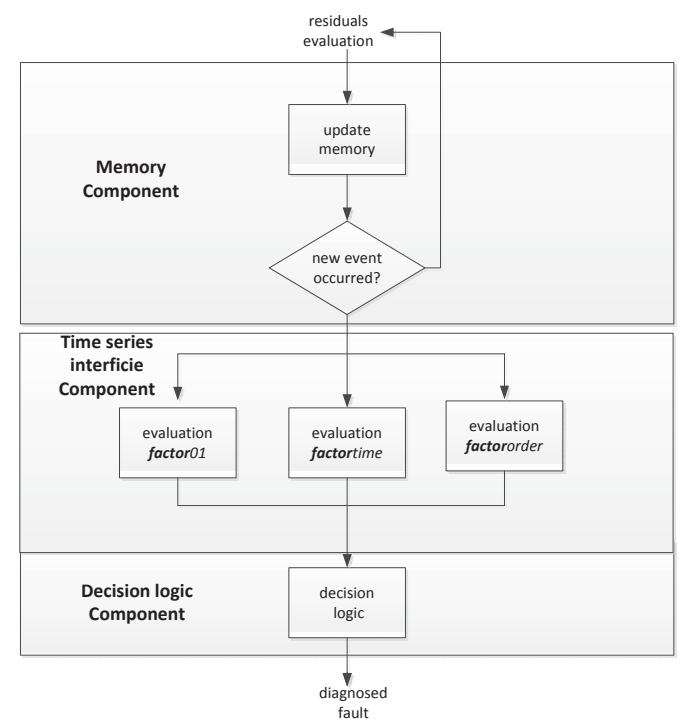

Fig. 3: Components of the interface and fault isolation modules.

because just the activation peaks are stored. If the fault detection component detects a new residual event $\left(\right.$ i.e. $\left.\phi_{i}(t)=1\right)$, the memory component table is updated by adding a new row that contains all previous event information. The problem of different time instant appearance of fault signals $\phi_{i}(t)$ is solved by not indicating the isolation decision until a prefixed waiting time $T_{w}$ has elapsed from the first fault signal appearance. This $T_{w}$ is calculated from the largest transient time response $T_{l t}$ from non-faulty situation to any faulty situation. After this time has elapsed, a diagnosis is proposed and the memory component is reset, being ready to start the diagnosis of a new fault. Inside this diagnosis window, the maximum activation value of the memory-table $\phi_{i, \max }$ corresponding to residual $i$ only changes if the current activation value $\phi_{i}\left(t_{0}\right)$ is superior to the previous ones according to (9).

\section{Pattern comparison component}

The pattern comparison component compares the memory contents with the stored fault patterns. Given a set of residuals $r_{1}, \ldots, r_{n r}$ and the considered set of faults $f_{1}, f_{2}, \ldots, f_{j}, \ldots, f_{m}$, each $r_{i}$ is affected by a subset of these faults. The fault patterns are organized according to a theoretical fault signature matrix named $F S M$. An element $F S M_{i j}$ of the matrix contains the pattern if $f_{j}$ is expected to affect $r_{i}$, otherwise it is equal to 0 . This interpretation assumes that the occurrence of $f_{j}$ is observable in $r_{i}$, hypothesis known as fault exoneration or no compensation, and that $f_{j}$ is the only fault affecting the monitored system. Three different fault signature matrices are considered in the evaluation task: Boolean fault signal activation (FSM01) that contains information about the incidence or no incidence of faults an residuals, fault signal occurrence order (FSMorder) that contains information about the expected order of theoretical incidence of a fault in the different residuals and time (FSMtime) that contains the information of the delay between the the first residual fault activation and the following residual fault activations. These matrices can be obtained from a fault sensitivity analysis of residuals.

\section{Fault diagnosis in the $\mathrm{CFr}$ system}

In the CFr system described in Section II, two different residuals $r_{C}(t)$ and $r_{F}(t)$ can be generated from the difference between the available level measurements in Cuinchy and Fontinettes $\left(y_{C}(t)\right.$ and $\left.y_{F}(t)\right)$ and the level estimations $\left(\hat{y}_{C}(t)\right.$ and $\left.\hat{y}_{F}(t)\right)$ that can be computed using model (2) with

$$
\hat{y}_{C}(t)=\mathscr{L}^{-1}\{y(0, s)\} \text { and } \hat{y}_{F}(t)=\mathscr{L}^{-1}\{y(L, s)\}
$$

and considering

$$
q(0, s)=\mathscr{L}\left\{q_{C}(t)\right\} \text { and } q(L, s)=\mathscr{L}\left\{q_{F}(t)\right\},
$$

where $q_{C}(t)$ and $q_{F}(t)$ are the flows in Cuinchy and Fontinettes, respectively. They can be computed with the known lock operation profiles $q \operatorname{lock} k_{C}(t)$ and $q \operatorname{lock} k_{F}(t)$ and the control gate flow in Cuinchy $u_{C}(t)$ (known control input).

The possible faults that have been considered in this work are sensor faults in both level sensors $f_{y_{C}}$ and $f_{y_{F}}$ and actuator faults in the Cuinchy control gate $f_{u_{C}}$. The effect of the considered faults in the different variables involved in the two residual computations is:

$$
\begin{aligned}
& y_{C}(t)=y_{C}^{0}(t)+f_{y_{C}}(t) \\
& y_{F}(t)=y_{F}^{0}(t)+f_{y_{F}}(t) \\
& q_{C}(t)=\operatorname{qlock}_{C}(t)+u_{C}(t)=\operatorname{qlock}_{C}(t)+u_{C}^{0}(t)+f_{u_{C}}(t) \\
& q_{F}(t)=\operatorname{qlock}_{F}(t)
\end{aligned}
$$

where $y_{C}^{0}(t), y_{F}^{0}(t)$ and $u_{C}^{0}(t)$ denote actual values of levels and control gate flow, respectively.

Then, considering the effect of the three faults in the two residuals, matrices FSM01 (Table I), FSMorder (Table II) and FSM time (Table III) can be obtained. Level sensor faults $f_{y_{C}}$ and $f_{y_{F}}$ only affect the associated level residual. So FSMorder matrix does not provide any additional information to the FSM01 matrix and neither does the FSMtime matrix, where $[-1,-1]$ denotes no influence of a fault in a residual as proposed in [17]. On the other hand, $f_{u_{C}}$ affects the two level residuals, first $r_{C}$ and later $r_{F}$. The time values $\underline{\tau}_{2,3}$ and $\bar{\tau}_{2,3}$ denote the minimum and maximum delays from the activation of the fault signal in $r_{C}$ to the activation of $r_{F}$ in the presence of an actuator fault in the Cuinchy control gate $f_{u_{C}}$. Therefore, when the fault signal associated to the Cuinchy level residual is activated, a waiting time $T_{w}=\bar{\tau}_{2,3}$ has to be considered in (9) to distinguish between a fault in the Cuinchy level sensor $\left(f_{y_{C}}\right)$ and an actuator fault in the Cuinchy control gate $\left(f_{u_{C}}\right)$. The values of $\underline{\tau}_{2,3}$ and $\bar{\tau}_{2,3}$ will be around the delay of the transfer function $\hat{p}_{12}^{\prime}(s)$ (i.e. $6930 s$ ).

\begin{tabular}{|c|c|c|c|}
\hline & $f_{y_{C}}$ & $f_{y_{F}}$ & $f_{u_{C}}$ \\
\hline$r_{C}$ & 1 & 0 & 1 \\
\hline$r_{F}$ & 0 & 1 & 1 \\
\hline
\end{tabular}

TABLE I: FSM01 matrix in the CFr system

\begin{tabular}{|c|c|c|c|}
\hline & $f_{y_{C}}$ & $f_{y_{F}}$ & $f_{u_{C}}$ \\
\hline$r_{C}$ & 1 & 0 & 1 \\
\hline$r_{F}$ & 0 & 1 & 2 \\
\hline
\end{tabular}

TABLE II: FSMorder matrix in the CFr system 


\begin{tabular}{|c|c|c|c|}
\hline & $f_{y_{C}}$ & $f_{y_{F}}$ & $f_{Q_{C}}$ \\
\hline$r_{C}$ & 0 & {$[-1,-1]$} & 0 \\
\hline$r_{F}$ & {$[-1,-1]$} & 0 & {$\left[\underline{\tau}_{2,3}, \bar{\tau}_{2,3}\right]$} \\
\hline
\end{tabular}

TABLE III: FSMtime matrix in the CFr system

\section{RESUlTs}

The IDZ models given in (5) are used to compute the results.

On the other hand, in order to cope with errors due to uncertainty in transport delays that are present in open-flow canal systems [18], following the ideas in [19], [20], the discrete residual has been computed as

$$
r(t)=y(t)-\hat{y}\left(t-\Delta \tau^{0}\right),
$$

where

$$
\Delta \tau^{0}=\arg \min _{\Delta \tau \in\left[-\lambda_{\tau}, \lambda_{\tau}\right]}|y(t)-\hat{y}(t-\Delta \tau)|,
$$

with $\lambda_{\tau}$ the maximum deviation from the nominal time delay.

Uncertainties in time delay produce important instantaneous errors in level estimations. Figure 4 shows the evolution of residuals computed directly with (6) and applying (13) in a realistic scenario. Maximum and minimum residual values in fault free scenarios have been chosen as residual bounds $\underline{\sigma}$ and $\bar{\sigma}$ used in the fault detection procedure (8). The residual bounds for the two residuals computed directly and applying (13) considering $\lambda_{\tau}=120 \mathrm{~s}$ in (14) are summarized in Table IV.

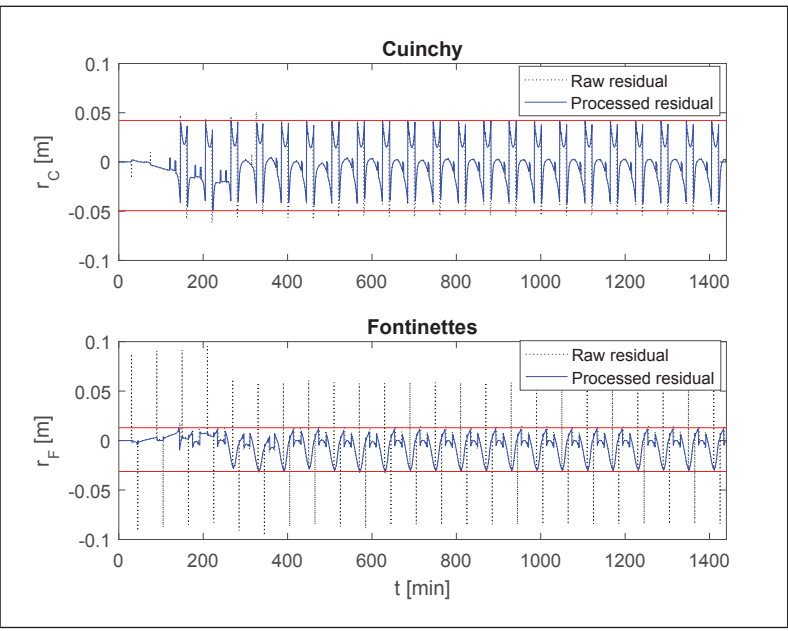

Fig. 4: Level residuals $r_{C}(t)$ and $r_{F}(t)$ in a 24-hour fault-free scenario.

\begin{tabular}{|c|c|c|c|c|}
\hline & \multicolumn{2}{|c|}{ Directly } & \multicolumn{2}{c|}{ Applying $\Delta \tau$} \\
\hline & $\underline{\sigma}$ & $\bar{\sigma}$ & $\underline{\sigma}$ & $\bar{\sigma}$ \\
\hline$r_{C}[m]$ & -0.062 & 0.052 & -0.05 & 0.042 \\
\hline$r_{F}[m]$ & -0.096 & 0.096 & -0.032 & 0.013 \\
\hline
\end{tabular}

TABLE IV: Residual fault detection thresholds

Different realistic fault scenarios have been generated in order to study the performance of the proposed fault diagnosis method considering FSM01 (Table I), FSMorder (Table II) and FSM time (Table III) with $\underline{\tau}_{2,3}=5900 \mathrm{~s}$ and $\bar{\tau}_{2,3}=7900 \mathrm{~s}$.
With the purpose of avoiding that uncertainty and modeling errors compensate the fault effects, when a fault is detected using (8), the residual is computed using (13) by considering $\Delta \tau^{0}$ computed with (14), but changing $\min$ by $\max$. In the following, the results of two fault scenarios are explained in detail.

Fault scenario 1: Additive fault in the Fontinettes level sensor

An additive fault of $6 \mathrm{~cm}$ is simulated at the Fontinettes level sensor at $t \geq 500 \mathrm{~min}$, which emulates a fall of $6 \mathrm{~cm}$ of an ultrasonic sensor from its support arm. Figure 5 shows the evolution of the two residuals: $r_{C}(t)$ is activated when the fault is produced (at $t=500 \mathrm{~min}$ ). Then, according to Tables I and II, the Fontinettes sensor fault is correctly isolated.

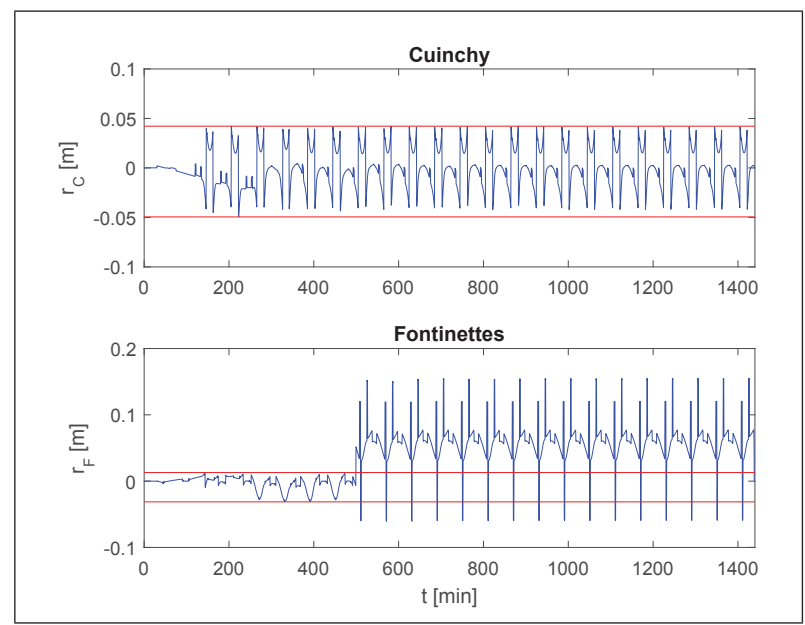

Fig. 5: Water level residuals $r_{C}(t)$ and $r_{F}(t)$ in a sensor fault scenario: Fontinettes level fault $f_{y_{F}}(t)=6 \mathrm{~cm}$ at $t \geq 500$ min

\section{Fault scenario 2: Additive fault in Cuinchy control gate}

An additive fault of $-4 \mathrm{~m}^{3} / \mathrm{s}$ is simulated at the Cuinchy control gate at $t \geq 300 \mathrm{~min}$ that emulates a partial obstruction in this gate. Figures 6 and 7 show the residuals and the fault signals evolution, respectively. The first fault signal $\phi_{C}(t)$ is activated at $t=342 \mathrm{~min}$ (42 $\mathrm{min}$ after the gate is partially blocked). Later, at $t=442 \mathrm{~min}$, the fault signal $\phi_{F}(t)$ is activated. Then, during $100 \mathrm{~min}$ (from $t=342 \mathrm{~min}$ to $t=442 \mathrm{~min})$ there are two fault candidates $\left(f_{y_{C}}\right.$ and $f_{u_{C}}$ ), according to Table I. However, after the activation of $\phi_{F}(t)$, only $f_{u_{C}}$ is consistent with the observed fault signals, according to Table II. The fault diagnosis procedure works despite the signal faults are intermittently activated, thanks to the memory component (9) and Table III.

\section{CONCLUSION}

A diagnosis method was proposed in this paper to detect and isolate sensor and actuator faults that can occur in flat navigation canals. Fault diagnosis in these systems is required to avoid the navigation stoppage. Indeed, the navigation is allowed only if the water level of each canal is inside a navigation rectangle of some centimeters around the Normal Navigation Level. The proposed diagnosis method was 


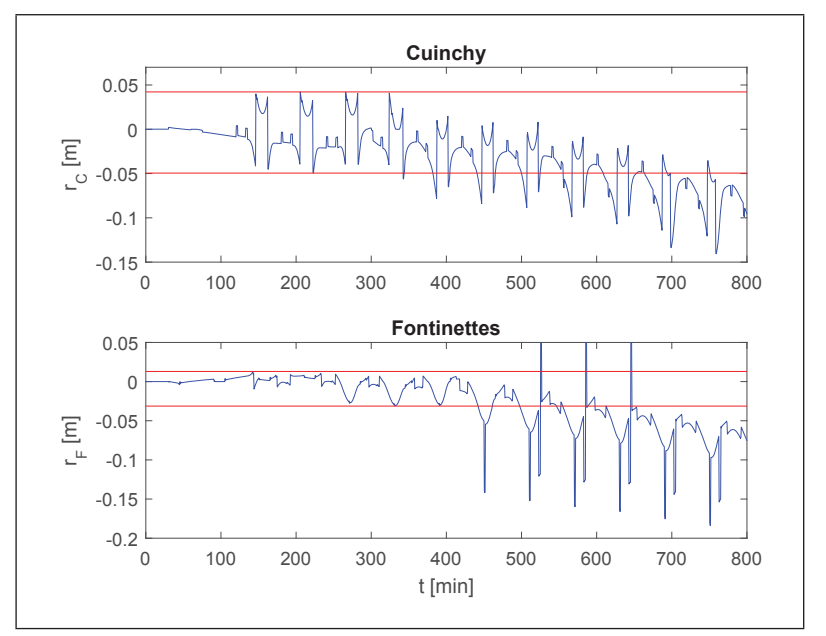

Fig. 6: Level residuals $r_{C}(t)$ and $r_{F}(t)$ in an actuator fault scenario: Cuinchy fault $f_{u_{C}}(t)=-4 \mathrm{~m}^{3} / \mathrm{s}$ at $t \geq 300 \mathrm{~min}$

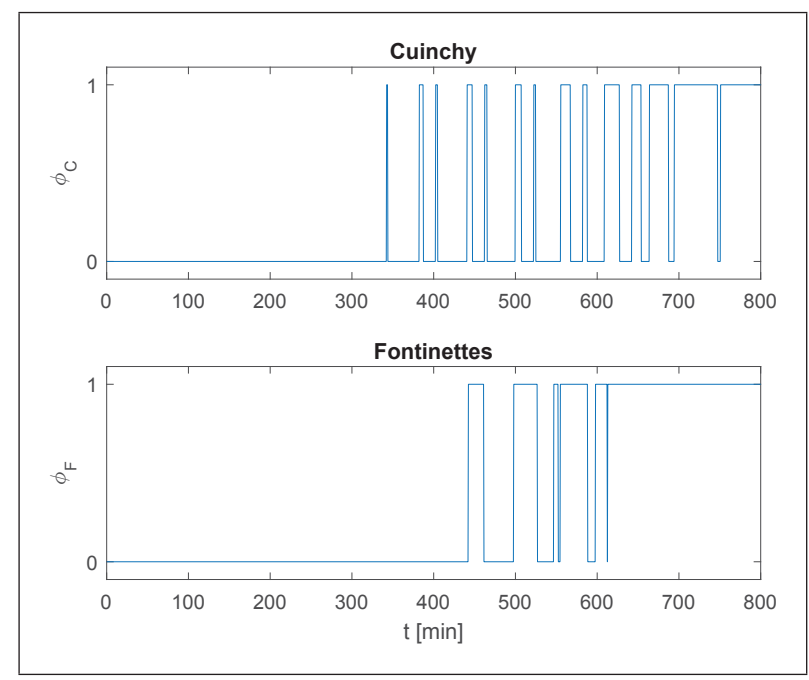

Fig. 7: Instantaneous activation signals $\phi_{C}(t)$ and $\phi_{F}(t)$ in an actuator fault in Cuinchy $\left(f_{u_{C}}(t)=-4 \mathrm{~m}^{3} / \mathrm{s}\right.$ at $\left.t \geq 300 \mathrm{~min}\right)$

designed based on the Integrator Delay Zero model, which is able to reproduce the peaks introduced by the significant lock operations. The Cuinchy-Fontinettes navigation reach that is located in the north of France was considered to test the proposed modeling and fault diagnosis approaches. Two fault scenarios based on realistic operating conditions were built to highlight the performance of the proposed diagnosis approach, and faults on sensors and actuators were correctly detected. In future works, multiple fault occurrences will be considered. In addition, the proposed diagnosis approach will be improved to deal with strong disturbances that characterize real environmental systems.

\section{ACKNOWLEDGMENT}

This work has been partially funded by the Spanish Ministry of Economy and Competitiveness (MINECOP) and FEDER through the project HARCRICS (ref. DPI2014-58104R) and through the grant IJCI-2014-2081.

\section{REFERENCES}

[1] V. T. Chow, "Open-channel hydraulics.” McGraw-Hill. New York., 1959.

[2] Y. Bolea, V. Puig, and J. Blesa, "Linear parameter varying modeling and identification for real-time control of open-flow irrigation canals," Environmental Modelling and Software, vol. 53, pp. 87-97, 2014.

[3] J. Schuurmans, A. J. Clemmens, S. Dijkstra, A. Hof, and R. Brouwer, "Modeling of irrigation and drainage canals for controller design," Journal of irrigation and drainage engineering, vol. 125, no. 6, pp. 338-344, 1999.

[4] X. Litrico and V. Fromion, "Simplified modeling of irrigation canals for controller design," Journal of Irrigation and Drainage Engineering, pp. 373-383, 2004.

[5] P. van Overloop, I. Miltenburg, X. Bombois, A. Clemmens, R. Strand, N. van de Giesen, and R. Hut, "Identification of resonance waves in open water channels," Control Engineering Practice, Volume 18, Issue 8, August, pp. 863-872, 2010.

[6] E. Duviella, L. Bako, M. Sayed-Mouchaweh, J. Blesa, Y. Bolea, V. Puig, and K. Chuquet, "Inland navigation channel model: Application to the Cuinchy-Fontinettes reach," in Networking, Sensing and Control (ICNSC), 2013 10th IEEE International Conference on. IEEE, 2013, pp. 164-169.

[7] K. Horváth, E. Duviella, J. Blesa, L. Rajaoarisoa, Y. Bolea, V. Puig, and K. Chuquet, "Gray-box model of inland navigation channel: application to the Cuinchy-Fontinettes reach," Journal of Intelligent Systems, vol. 23, no. 2, pp. 183-199, 2014.

[8] J. Blesa, K. Horváth, E. Duviella, V. Puig, Y. Bolea, L. Rajaoarisoa, and $\mathrm{K}$. Chuquet, "Model-based sensor supervision in inland navigation networks: Cuinchy-fontinettes case study," Journal of Maritime Research, vol. 11, no. 2, pp. 81-88, 2014.

[9] K. Horváth, J. Blesa, E. Duviella, L. Rajaoarisoa, V. Puig, and K. Chuquet, "Sensor fault diagnosis of inland navigation system using physical model and pattern recognition approach," IFAC Proceedings Volumes, vol. 47, no. 3, pp. 5309-5314, 2014, 19th IFAC World Congress.

[10] E. Duviella, L. Rajaoarisoa, J. Blesa, and K. Chuquet, "Fault detection and isolation of inland navigation channel: Application to the CuinchyFontinettes reach," in 52nd IEEE Conference on Decision and Control, Florence, Firenze, Italy, December 10-13, 2013, pp. 4877-4882.

[11] P.-O. Malaterre, D. Dorchies, and J.-P. Baume, "Automatic tuning of robust PI controllers for a cascade of rivers or irrigation canals pools," European Control Conference, Strasbourg, France, June 24-27, 2014.

[12] J. Gertler, Fault Detection and Diagnosis in Engineering Systems. New York: Marcel Dekker, 1998.

[13] M. Blanke, M. Kinnaert, J. Lunze, and M. Staroswiecki, Diagnosis and fault-tolerant control. Springer-Verlag Berlin Heidelberg, 2006.

[14] R. Isermann, Fault Diagnosis Systems: An Introduction from Fault Detection to Fault Tolerance. Springer, New York, 2006.

[15] J. Chen and R. J. Patton, Robust model-based fault diagnosis for dynamic systems. Kluwer Academic Publishers, 1999.

[16] J. Meseguer, V. Puig, and T. Escobet, "Fault diagnosis using a timed discrete-event approach based on interval observers: Application to sewer networks," IEEE Transactions on Systems, Man, and Cybernetics Part A:Systems and Humans, vol. 40, no. 5, pp. 900-916, 2010.

[17] V. Puig and J. Blesa, "Limnimeter and rain gauge FDI in sewer networks using an interval parity equations based detection approach and an enhanced isolation scheme," Control Engineering Practice, vol. 21, no. 2, pp. 146-170, 2013.

[18] Y. Bolea, V. Puig, and J. Blesa, "Gain-scheduled Smith predictor PIDbased LPV controller for open-flow canal control," IEEE Transactions on Control Systems Technology, vol. 22, no. 2, pp. 468-477, 2014.

[19] J. Blesa, V. Puig, and Y. Bolea, "Fault detection using interval LPV models in an open-flow canal," Control Engineering Practice, vol. 18, no. 5, pp. 460-470, 2010.

[20] J. Blesa, P. Jiménez, D. Rotondo, F. Nejjari, and V. Puig, "An interval NLPV parity equations approach for fault detection and isolation of a wind farm," IEEE Transactions on Industrial Electronics, vol. 62, no. 6, pp. 3794-3805, 2015. 